\title{
Buckling analysis of sandwich orthotropic cylindrical shells by considering the geometrical imperfection in face sheets
}

\author{
S. A. Ahmadi ${ }^{a}$, M. H. Pashaei ${ }^{a, *}$, R.-A. Jafari-Talookolaei ${ }^{a}$ \\ ${ }^{a}$ School of Mechanical Engineering, Babol Noshirvani University of Technology, Shariati Ave., 47148-71167, Babol, Mazandaran, Iran
}

Received 8 June 2018; accepted 13 February 2019

\begin{abstract}
A three dimensional analysis for the buckling behavior of a sandwich orthotropic thick cylindrical shell under uniform lateral pressure has been presented. It is assumed that both ends of the shell have the simply supported conditions and the face sheets have an axisymmetric initial geometrical imperfection in the axial direction. The governing differential equations are derived based on the second Piola-Kirchhoff stress tensor and are reduced to a homogenous linear system of equations using differential quadrature method (DQM). The buckling pressures have been calculated for the shell with isotropic core and orthotropic face sheets with 0-degree orientation with respect to the hoop direction. Moreover, buckling pressure reduction parameter has been defined and computed for different imperfection parameters of face sheets and geometrical properties of sandwich shells. The results obtained in the present work are compared with finite element solutions and results reported in the literature and very good agreements have been observed. It is shown that the imperfections have higher effects on the buckling load of thick shells than thin ones. Likewise, it is found that the present method can capture the various geometrical imperfections observed during the manufacturing process or service life.
\end{abstract}

(c) 2019 University of West Bohemia. All rights reserved.

Keywords: thick sandwich shell, orthotropic materials, buckling pressure, geometrical imperfections, differential quadrature method

\section{Introduction}

The Lightweight and durable structures such as sandwich cylindrical shells are one of the main requirements for aerospace industry, marine structures, ground transportation and airplane vehicle to enhance their structural efficiency and performance. These types of shells that consist of two high stiffness thin faces, which are separated by a low density thick core, provide efficient structural design solutions due to the high strength/stiffness to weight ratio. In applications with compressive loading, the loss of stability and the accurate prediction of buckling loads of such structures is one of the primary concerns. This is particularly important in sandwich shells because of their low bending stiffness in core. In addition, the sandwich composite shells are normally used as the thick structure and thus the thickness effects need to be properly investigated.

The buckling behavior of sandwich cylindrical shells has been studied by many researchers. Elasticity solutions for monolithic homogeneous orthotropic cylindrical shells have been provided by Lekhnitskii [12] and Kardomateas [18]. After that, elasticity solutions for sandwich shells have been obtained by properly extending the solutions for monolithic structures [13]. Kardomateas and simitses [15] have presented a theoretical prediction of buckling loads of sandwich long cylindrical shells with laminated facings and foam core under the action of uniform

\footnotetext{
*Corresponding author. Tel.: +981 132332 072, e-mail: mpashaei@nit.ac.ir. https://doi.org/10.24132/acm.2019.460
} 
hydrostatic pressure. They have used the three-dimensional elasticity to obtain the response and the results have been generated with and without considering the transverse shear effect. Kardomateas [14] has investigated the buckling behavior of thick composite and sandwich cylindrical shells. In this work, the three dimensional elasticity solutions based on the Piola-Kirchhoff stress tensor have been used and the results have been compared against some other shell theories. Arjomandi and Taheri $[2,3]$ have presented the analytical and finite element solutions for the buckling analysis of the sandwich pipes having various structural configurations and core materials under the action of external pressure. In these studies, the buckling behavior of the pipes has been investigated using a two-dimensional formulation in the polar coordinate system. An approximate analytical solution for the buckling of the composite sandwich cylindrical shell with clamped ends under uniform external pressure has been presented by Lopatin and Morozov [19]. Ohga et al. [25] have applied the reduced stiffness lower bound method to solve the buckling problem of the sandwich cylindrical shell with simply supported ends. Stability of long cylindrical sandwich shells with different facings subjected to a lateral external pressure has been carried out by Birman and Simitres [6]. The state of plane strain has been assumed to model the shell. Malikan et al. [23] used a nonlocal three-dimensional theory of elasticity in order to studied buckling behavior of functionally graded porous nanoplates.

In the early of 1930, researchers have performed several experimental studies on the buckling behavior of shells $[10,20]$. It is shown that there are great scatter in experimental results and the experimental values have been obtained much lower than the theoretical ones. It is found that this is due to the imperfection which is occurred in the shell. This led to the establishment of some reliable and modified guidelines based on the experimental results $[8,24]$. Nowadays, the non-destructive methods have attracted great interest among researchers to evaluate the actual buckling behaviors of cylindrical shells considering various geometrical imperfections $[9,16,26]$. Based on the reported literatures, it is concluded that the various approaches and guidelines which have been developed to predict realistic buckling loads have a lot of limitations in the shape of structure and their accuracy are dependent on the type and amplitude of imperfection. This highlights the need for a methodology to be able to consider the effects of any type of imperfection without the need for the expensive experimental examination. An exprimental and numerical buckling analysis was carried out by Wang et al. [29] on the quasi-perfect cylindrical shell under axial compression. The effects of initial axisymmetric imperfection on the thermal buckling of cylindrical shell with FGM coating has been investigated by Wang et al. [32].

Due to the complexity of the sandwich shells, numerical and semi-analytical methods are usually used to obtain the solutions for any combinations of boundary, continuity and loading condition. Due to its simplicity, accuracy, and computational efficiency, DQM is one of the semi-analytical methods that frequently used to analyze the sandwich structures. This method generally needs low grid points to achieve results with good accuracy. Wang and Yuan [31] have used DQM to study stress analysis of sandwich panels. Static analysis of sandwich panels with non-homogeneous soft cores has been carried out by Wang and Yuan [30]. Milikan and Safraee Far [22] used differential quadrature method to investigate dynamic buckling of graphene sheet coupled by a viscoelastic medium. In this work the neperian frequency based on nonlocal elasticity theory was applied and non-linear quations of motion were obtained using Mindlin plate theory. They reported that when nanoscale parameters give great values, the small scale had further influences on the dynamic buckling of sheets. Maleki and Ahmadi [21] studied buckling behavior of the orthotropic thick cylindrical panel with geometrical imperfection using DQM. 
In this paper and for the first time, the equations for the buckling of sandwich orthotropic thick cylindrical shell under the action of uniform lateral pressure have been obtained based on the three dimensional benchmark elasticity solutions. An axisymmetric imperfection for the face sheets in axial direction has been considered in the formulations. Using DQM, the governing differential equations have been discretized and reduced to a system of linear homogenous equations. Numerical results have been obtained for sandwich shells with isotropic foam core and composite face sheet. The results are compared against the results obtained from commercial software ANSYS and those reported in the literatures. The presented method can be used to evaluate the buckling pressure of any types of sandwich orthotropic cylindrical shells by considering the various types of geometrical imperfections in the face sheets.

\section{Problem formulation}

Fig. 1 shows a cylindrical sandwich shell with length $L$, core thickness $h_{c}$, outer and inner face sheets thickness $h_{o}$ and $h_{i o}$, outer and inner radius $R_{2}$ and $R_{1}$ and mid-radius $a=\left(R_{1}+R_{2}\right) / 2$, respectively. A coordinate system $(r, \theta, x)$ is placed at one end of the shell and its origin is placed at the center of the shell. It is assumed that the shell is simply supported at both ends and lateral pressure $(P)$ is considered to act on the outer face of the shell. The displacement components in the axial $(x)$, circumferential $(\theta)$ and radial $(r)$ directions are denoted by $(u, v, w)$, respectively. The core of the sandwich shell is made up isotropic material such as alloy foam. Likewise, for the face sheets the unidirectional layer made up carbon/epoxy or glass/epoxy with 0-degree orientation with respect to the hoop direction are considered.
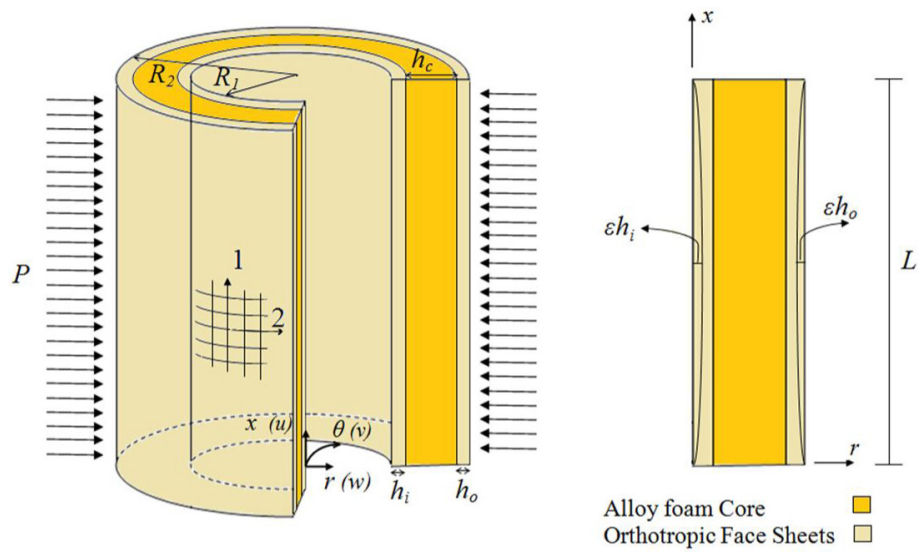

Fig. 1. Schematic view of the thick cylindrical sandwich shell

In most of structures, an inevitable differences in the thickness characteristics caused by imperfections that are usually produced in manufacturing process or developed in corrosive environments are expected to be present. In this paper assumed that the imperfection in the sandwich panel face sheets thicknesses vary trigonometrically in the axial direction. Variation of the thickness and the imperfection of the facesheets are shown in the right side of Fig. 1. In order to consider this types of geometrical imperfection, the thickness of the face sheets is defined as the function of $x$ as follows:

$$
h_{i, o}(x)=h 0_{i, o}\left(1-\varepsilon_{i, o} \cdot \cos \frac{\pi}{L}\left(x-\frac{L}{2}\right)\right),
$$

where $h 0_{i, o}$ is the thickness of the face sheets in perfect conditions and $\varepsilon_{i, o}$ is the imperfection parameter. According to the presented formulation, the mid-surface radius $h_{i, o}(x)$ varies in the 
axial direction while the inner radius $R_{1}$ is assumed to be constant. Also thicknesses of the panel at two ends are the same for all values of $\varepsilon$, i.e. $h(x)=h_{0}$ at $x=0, L$ and $h(x)=h_{0}(1-\varepsilon)$ at $x=L / 2$. The core thickness is assumed to be constant.

In this work, the stability equations have been separately obtained for the core and two face sheets. These equations have been solved by considering the traction and displacement continuity at the face sheet and core interfaces. The derivation is similar to that presented by Akbari Alashti and Ahmadi [1] except that we have included the orthotropic behavior for face sheets in the formulations. According to this method, displacement fields for the shells are expressed by two terms consist of primary and infinitely perturbed positions:

$$
\begin{aligned}
w(r, \theta, x) & =w^{0}(r, \theta, x)+\alpha \cdot w^{\prime}(r, \theta, x), \\
v(r, \theta, x) & =v^{0}(r, \theta, x)+\alpha \cdot v^{\prime}(r, \theta, x), \\
u(r, \theta, x) & =u^{0}(r, \theta, x)+\alpha \cdot u^{\prime}(r, \theta, x),
\end{aligned}
$$

where $\alpha$ is an infinitesimally small quantity, $w^{0}(r, \theta, x), v^{0}(r, \theta, x), u^{0}(r, \theta, x)$ are components of the displacement field corresponding to the primary position and $w^{\prime}(r, \theta, x), v^{\prime}(r, \theta, x)$ and $u^{\prime}(r, \theta, x)$ denote the values of components of displacement field in the disturbed position in the radial, circumferential and axial directions, respectively. The orthotropic stress-strain relations are as follows:

$$
\left[\begin{array}{l}
\sigma_{11} \\
\sigma_{22} \\
\sigma_{33} \\
\tau_{23} \\
\tau_{31} \\
\tau_{12}
\end{array}\right]=\left[\begin{array}{cccccc}
C_{11} & C_{12} & C_{13} & 0 & 0 & 0 \\
C_{12} & C_{22} & C_{23} & 0 & 0 & 0 \\
C_{13} & C_{23} & C_{33} & 0 & 0 & 0 \\
0 & 0 & 0 & C_{44} & 0 & 0 \\
0 & 0 & 0 & 0 & C_{55} & 0 \\
0 & 0 & 0 & 0 & 0 & C_{66}
\end{array}\right]\left[\begin{array}{l}
\varepsilon_{11} \\
\varepsilon_{22} \\
\varepsilon_{33} \\
\varepsilon_{23} \\
\varepsilon_{31} \\
\varepsilon_{12}
\end{array}\right]
$$

Here $C_{i j}$ denote the stiffness constants and can be written in terms of material constants as below:

$$
\begin{aligned}
C_{11} & =\frac{1-\nu_{23} \cdot \nu_{32}}{E_{22} E_{33} \Delta}, \quad C_{12}=\frac{\nu_{21}-\nu_{31} \cdot \nu_{23}}{E_{22} E_{33} \Delta}, \\
C_{22} & =\frac{1-\nu_{13} \cdot \nu_{31}}{E_{11} E_{33} \Delta}, \quad C_{13}=\frac{\nu_{31}-\nu_{21} \cdot \nu_{32}}{E_{11} E_{33} \Delta}, \\
C_{33} & =\frac{1-\nu_{12} \cdot \nu_{21}}{E_{22} E_{11} \Delta}, \quad C_{23}=\frac{\nu_{32}-\nu_{12} \cdot \nu_{31}}{E_{22} E_{11} \Delta}, \\
C_{44} & =G_{23}, \quad C_{55}=G_{31}, \quad C_{66}=G_{12}, \\
\Delta & =\frac{1-\nu_{12} \cdot \nu_{21}-\nu_{23} \cdot \nu_{32}-\nu_{13} \cdot \nu_{31}-2 \nu_{21} \cdot \nu_{32} \cdot \nu_{13}}{E_{11} E_{22} E_{33}} .
\end{aligned}
$$

Moreover, for the core materials, the stress-strain relations can be written as follows:

$$
\begin{array}{ll}
\sigma_{r r}=(2 G+\lambda) \varepsilon_{r r}+\lambda\left(\varepsilon_{\theta \theta}+\varepsilon_{x x}\right), & \tau_{r \theta}=G \varepsilon_{r \theta}, \\
\sigma_{\theta \theta}=(2 G+\lambda) \varepsilon_{\theta \theta}+\lambda\left(\varepsilon_{x x}+\varepsilon_{r r}\right), & \tau_{r x}=G \varepsilon_{r x}, \\
\sigma_{x x}=(2 G+\lambda) \varepsilon_{x x}+\lambda\left(\varepsilon_{\theta \theta}+\varepsilon_{r r}\right), & \tau_{x \theta}=G \varepsilon_{x \theta},
\end{array}
$$

where $\lambda$ and $G$ are the Lame coefficients for foam materials. Likewise, the strain-displacement 
and rotation-displacement relations are:

$$
\begin{gathered}
\varepsilon_{r r}=\frac{\partial w}{\partial r}, \quad \varepsilon_{r x}=\frac{1}{2}\left(\frac{\partial w}{\partial x}+\frac{\partial u}{\partial r}\right), \\
\varepsilon_{x x}=\frac{\partial u}{\partial x}, \quad \varepsilon_{r \theta}=\frac{1}{2}\left(\frac{1}{r} \frac{\partial w}{\partial \theta}+\frac{\partial v}{\partial r}-\frac{v}{r}\right), \\
\varepsilon_{\theta \theta}=\frac{1}{r} \frac{\partial v}{\partial \theta}+\frac{w}{r}, \quad \varepsilon_{\theta x}=\frac{1}{2}\left(\frac{1}{r} \frac{\partial u}{\partial \theta}+\frac{\partial v}{\partial x}\right) \\
\omega_{r x}=\frac{1}{2}\left(\frac{\partial w}{\partial x}-\frac{\partial u}{\partial r}\right) \\
\omega_{\theta x}=\frac{1}{2}\left(\frac{1}{r} \frac{\partial u}{\partial \theta}-\frac{\partial v}{\partial x}\right), \\
\omega_{r \theta}=-\frac{1}{2}\left(-\frac{1}{r} \frac{\partial w}{\partial \theta}+\frac{\partial v}{\partial r}+\frac{v}{r}\right) .
\end{gathered}
$$

The stress and strain components in the buckled state can be expressed as:

$$
\begin{array}{ll}
\varepsilon_{r r}(r, \theta, x)=\varepsilon_{r r}^{0}(r, \theta, x)+\alpha \cdot \varepsilon_{r r}^{\prime}(r, \theta, x), & \gamma_{r \theta}(r, \theta, x)=\gamma_{r \theta}^{0}(r, \theta, x)+\alpha \cdot \gamma_{r \theta}^{\prime}(r, \theta, x), \\
\varepsilon_{x x}(r, \theta, x)=\varepsilon_{x x}^{0}(r, \theta, x)+\alpha \cdot \varepsilon_{x x}^{\prime}(r, \theta, x), & \gamma_{r x}(r, \theta, x)=\gamma_{r x}^{0}(r, \theta, x)+\alpha \cdot \gamma_{r x}^{\prime}(r, \theta, x), \\
\varepsilon_{\theta \theta}(r, \theta, x)=\varepsilon_{\theta \theta}^{0}(r, \theta, x)+\alpha \cdot \varepsilon_{\theta \theta}^{\prime}(r, \theta, x), & \gamma_{x \theta}(r, \theta, x)=\gamma_{x \theta}^{0}(r, \theta, x)+\alpha \cdot \gamma_{x \theta}^{\prime}(r, \theta, x), \\
\sigma_{r r}(r, \theta, x)=\sigma_{r r}^{0}(r, \theta, x)+\alpha \cdot \sigma_{r r}^{\prime}(r, \theta, x), & \left.\tau_{r \theta}(r, \theta, x)=\tau_{r \theta}^{0}(r, \theta, x)+\alpha \cdot \tau_{r \theta}^{\prime} r, \theta, x\right), \\
\sigma_{\theta \theta}(r, \theta, x)=\sigma_{\theta \theta}^{0}(r, \theta, x)+\alpha \cdot \sigma_{\theta \theta}^{\prime}(r, \theta, x), & \tau_{r x}(r, \theta, x)=\tau_{r x}^{0}(r, \theta, x)+\alpha \cdot \tau_{r x}^{\prime}(r, \theta, x), \\
\sigma_{x x}(r, \theta, x)=\sigma_{x x}^{0}(r, \theta, x)+\alpha \cdot \sigma_{x x}^{\prime}(r, \theta, x), & \tau_{x \theta}(r, \theta, x)=\tau_{x \theta}^{0}(r, \theta, x)+\alpha \cdot \tau_{x \theta}^{\prime}(r, \theta, x) .
\end{array}
$$

Based on the second Piola-Kirchhoff stress tensor $\sigma$, the three dimensional equations of equilibrium can be derived as [7]:

$$
\operatorname{div}\left(\boldsymbol{\sigma} \cdot \mathbf{F}^{T}\right)=0, \quad \mathbf{F}=\mathbf{I}+\operatorname{grad}(\vec{V}),
$$

where $\mathbf{F}$ is the deformation gradient, $\vec{V}$ is the displacement vector and $\mathbf{I}$ is the unit tensor. Considering linear normal strains, rotations, and stresses and keeping the linear terms in $\alpha$, we can obtain buckling equations of a monolithic cylindrical shell in radial, circumferential and axial directions. It is assumed that the non-zero normal strains be much smaller than 1 and the shear strain, shear stress and rotation can be neglected in the equilibrium conditions. After substituting the presented equations into (9) and followed considerations, a system of homogeneous differential equations is obtained in simplified forms as follow:

$$
\begin{gathered}
\frac{\partial}{\partial r}\left(\sigma_{r r}^{0} \varepsilon_{r r}^{\prime}+\sigma_{r r}^{\prime}\right)+\frac{1}{r} \frac{\partial}{\partial \theta}\left(\tau_{r \theta}^{\prime}+\sigma_{\theta \theta}^{0}\left(\varepsilon_{r \theta}^{\prime}+\omega_{r \theta}^{\prime}\right)\right)+ \\
\frac{\partial}{\partial x}\left(\tau_{r x}^{\prime}+\sigma_{x x}^{0}\left(\varepsilon_{r x}^{\prime}+\omega_{r x}^{\prime}\right)\right)+\frac{1}{r}\left(\sigma_{r r}^{\prime}+\sigma_{r r}^{0} \varepsilon_{r r}^{\prime}-\sigma_{\theta \theta}^{\prime}-\sigma_{\theta \theta}^{0} \varepsilon_{\theta \theta}^{\prime}\right)=0 \\
\frac{\partial}{\partial r}\left(\tau_{r \theta}^{\prime}+\sigma_{r r}^{0}\left(\varepsilon_{r \theta}^{\prime}-\omega_{r \theta}^{\prime}\right)\right)+\frac{1}{r} \frac{\partial}{\partial \theta}\left(\sigma_{\theta \theta}^{0} \varepsilon_{\theta \theta}^{\prime}+\sigma_{\theta \theta}^{\prime}\right)+ \\
\frac{\partial}{\partial x}\left(\tau_{\theta x}^{\prime}+\sigma_{x x}^{0}\left(\varepsilon_{\theta x}^{\prime}-\omega_{\theta x}^{\prime}\right)\right)+\frac{1}{r}\left(\sigma_{r r}^{0}\left(\varepsilon_{r \theta}^{\prime}-\omega_{r \theta}^{\prime}\right)+2 \tau_{r \theta}^{\prime}+\sigma_{\theta \theta}^{0}\left(\varepsilon_{r \theta}^{\prime}+\omega_{r \theta}^{\prime}\right)\right)=0, \\
\frac{\partial}{\partial r}\left(\tau_{r x}^{\prime}+\sigma_{r r}^{0}\left(\varepsilon_{r x}^{\prime}-\omega_{r x}^{\prime}\right)\right)+\frac{1}{r} \frac{\partial}{\partial \theta}\left(\sigma_{\theta \theta}^{0}\left(\varepsilon_{\theta x}^{\prime}+\omega_{\theta x}^{\prime}\right)+\tau_{\theta x}^{\prime}\right)+ \\
\frac{\partial}{\partial x}\left(\sigma_{x x}^{\prime}+\sigma_{x x}^{0} \varepsilon_{x x}^{\prime}\right)+\frac{1}{r}\left(\tau_{r x}^{\prime}+\sigma_{r r}^{0}\left(\varepsilon_{r x}^{\prime}-\omega_{r x}^{\prime}\right)\right)=0 .
\end{gathered}
$$




\section{S. A. Ahmadi et al. / Applied and Computational Mechanics 13 (2019) 5-20}

The corresponding boundary conditions of the sandwich shell are as follows:

(a) The boundary conditions in initial positions for two lateral surfaces due to the action of the lateral pressure $P$ can be presented using linear elasticity theory [17]:

$$
\sigma_{\theta \theta}^{0}=-P \frac{\left[1+\left(\frac{R_{1}}{r}\right)^{2}\right]}{\left[1-\left(\frac{R_{1}}{R_{2}}\right)^{2}\right]}=-P \cdot f_{\theta \theta}, \quad \sigma_{r r}^{0}=-P \frac{\left[1-\left(\frac{R_{1}}{r}\right)^{2}\right]}{\left[1-\left(\frac{R_{1}}{R_{2}}\right)^{2}\right]}=-P \cdot f_{r r} .
$$

(b) Six traction conditions at the bounding surfaces of the shells for the perturbed equilibrium positions have to be considered. These boundary conditions can be obtained by employing the equilibrium equations using the second Piola-Kirchhoff tensor $\sigma$ from equation (9) [7] as follows:

$$
{\sigma_{r r}^{\prime(1)}}_{R_{R_{1}, \theta}}={\sigma_{r r}^{\prime(3)}}_{R_{2}, \theta}=0,\left.\quad \tau_{r \theta}^{\prime(1)}\right|_{R_{1}, \theta}=\left.\tau_{r \theta}^{\prime(3)}\right|_{R_{2}, \theta}=0, \quad{\tau_{r x}^{\prime(1)}}_{R_{R_{1}, \theta}}=\left.\tau_{r x}^{\prime(3)}\right|_{R_{2}, \theta}=0,
$$

in which prime represent stress component in the perturbed positions and superscripts ${ }^{(1)}$ and

(3) denote the stress component at the inner and outer face sheets, respectively.

(c) Six compatibility conditions at the face sheets and core interfaces can be obtained in the following forms:

$$
\begin{aligned}
\left.\sigma_{r r}^{\prime(1)}\right|_{R_{1}+h_{i}, \theta} & =\left.{\sigma^{\prime}}_{r r}^{(2)}\right|_{R_{1}+h_{i}, \theta}, & \left.\sigma_{r r}^{\prime(2)}\right|_{R_{1}+h_{c}+h_{i}, \theta} & =\left.{\sigma_{r r}^{\prime}}_{r r}^{(3)}\right|_{R_{1}+h_{c}+h_{i}, \theta}, \\
\left.\tau_{r \theta}^{\prime(1)}\right|_{R_{1}+h_{i}, \theta} & =\left.\tau_{r \theta}^{\prime(2)}\right|_{R_{1}+h_{i}, \theta}, & \left.\tau_{r \theta}^{\prime(2)}\right|_{R_{1}+h_{c}+h_{i}, \theta} & =\left.{\tau^{\prime}}_{r \theta}^{(3)}\right|_{R_{1}+h_{c}+h_{i}, \theta}, \\
\left.\tau_{r x}^{\prime(1)}\right|_{R_{1}+h_{i}, \theta} & =\left.\tau_{r x}^{\prime(2)}\right|_{R_{1}+h_{i}, \theta}, & \left.\tau_{r x}^{\prime(2)}\right|_{R_{1}+h_{c}+h_{i}, \theta} & =\left.{\tau_{r x}^{\prime(3)}}_{r x}\right|_{R_{1}+h_{c}+h_{i}, \theta},
\end{aligned}
$$

in which superscript ${ }^{(2)}$ denotes the stress component at the core of the sandwich shell.

(d) Six displacement continuity conditions at the face sheets and core interfaces are as follows:

$$
\begin{aligned}
\left.w^{\prime(1)}(r, \theta, x)\right|_{R_{1}+h_{i}, \theta} & =\left.w^{\prime(2)}(r, \theta, x)\right|_{R_{1}+h_{i}, \theta}, \\
\left.v^{\prime(1)}(r, \theta, x)\right|_{R_{1}+h_{i}, \theta} & =\left.v^{\prime(2)}(r, \theta, x)\right|_{R_{1}+h_{i}, \theta}, \\
\left.u^{\prime(1)}(r, \theta, x)\right|_{R_{1}+h_{i}, \theta} & =\left.u^{\prime(2)}(r, \theta, x)\right|_{R_{1}+h_{i}, \theta}, \\
\left.w^{\prime(2)}(r, \theta, x)\right|_{R_{1}+h_{c}+h_{i}, \theta} & =\left.w^{\prime(3)}(r, \theta, x)\right|_{R_{1}+h_{c}+h_{i}, \theta}, \\
\left.v^{\prime(2)}(r, \theta, x)\right|_{R_{1}+h_{c}+h_{i}, \theta} & =\left.v^{\prime(3)}(r, \theta, x)\right|_{R_{1}+h_{c}+h_{i}, \theta}, \\
\left.u^{\prime(2)}(r, \theta, x)\right|_{R_{1}+h_{c}+h_{i}, \theta} & =\left.u^{\prime(3)}(r, \theta, x)\right|_{R_{1}+h_{c}+h_{i}, \theta} .
\end{aligned}
$$

(e) By assuming the simple end conditions for the shell, the following boundary conditions for the shell can be written:

$$
\begin{aligned}
& w^{\prime}=v^{\prime}=u^{\prime}=\frac{\partial^{2} w^{\prime}}{\partial x^{2}}=0 \quad \text { at } x=0 \\
& w^{\prime}=v^{\prime}=\sigma_{x x}^{\prime}=\frac{\partial^{2} w^{\prime}}{\partial x^{2}}=0 \quad \text { at } x=L .
\end{aligned}
$$




\section{The calculation of buckling load}

In this paper, the initial imperfection of the face sheet and the loading condition of the sandwich shell are assumed to be axisymmetric. By considering the boundary conditions (15), the perturbed displacements can be written in the following form:

$$
\begin{aligned}
w^{\prime}(r, \theta, x) & =B(r, x) \cdot \sin (m \theta), \\
v^{\prime}(r, \theta, x) & =A(r, x) \cdot \cos (m \theta), \\
u^{\prime}(r, \theta, x) & =C(r, x) \cdot \sin (m \theta),
\end{aligned}
$$

where $m$ is the buckling mode number in the circumferential direction. Substituting the equation (16) into (5) and (6), components of the stress field are expressed in terms of components of the displacement field. Substituting the above expression in (10), the buckling equations can be obtained in terms of the displacement field.

In the present work, the DQ method as a semi-analytical method, which has been introduced in reference [28], is used to discretize and solve the buckling equations. This method suggests that the first order derivative of the typical function $f(x, r)$ in radial and axial directions can be approximated as a linear sum of all functional values in the domain:

$$
\begin{aligned}
& \left.\frac{\partial f}{\partial r}\right|_{r=r_{i}}=\sum_{j=1}^{N} a_{i j}^{(1)} \cdot f\left(r_{j}, x\right) \quad \text { for } i=1,2, \ldots, N, \\
& \left.\frac{\partial f}{\partial x}\right|_{x=x_{i}}=\sum_{j=1}^{Q} c_{i j}^{(1)} \cdot f\left(r, x_{j}\right) \quad \text { for } i=1,2, \ldots, Q,
\end{aligned}
$$

where $a_{i j}^{(1)}$ and $c_{i j}^{(1)}$ are the weighting coefficients for the first derivative and $N$ and $Q$ denote the number of sampling points in $r$ and $x$ directions, respectively. The Chebyshev-Gauss-Lobatto points that have been shown [29] are more accurate than the equally spaced, is used. In this way, sampling points for radial and longitudinal direction can be wrriten as:

$$
\begin{aligned}
& r_{j}=\frac{r_{o}-r_{i}}{2}\left(1-\cos \frac{j-1}{N-1} \pi\right) \quad j=1, \ldots, N, \\
& x_{k}=\frac{L}{2}\left(1-\cos \frac{k-1}{Q-1} \pi\right) \quad k=1, \ldots, Q .
\end{aligned}
$$

There are different methods to evaluate the weighting coefficients [30]. In this paper, the polynomial expansion based on the differential quadrature is used to discretize the differential equations in the radial and longitudinal directions. Weighting coefficients for the first-order derivatives are defined based on the Lagrange interpolation polynomials as:

$$
c_{i j}^{(1)}=\frac{M^{(1)}\left(x_{i}\right)}{\left(x_{i}-x_{j}\right) \cdot M^{(1)}\left(x_{j}\right)}, \quad c_{i i}^{(1)}=\frac{M^{(2)}\left(x_{i}\right)}{2 M^{(1)}\left(x_{i}\right)} \quad \text { for } i \neq j,
$$

where

$$
\begin{aligned}
M^{(1)}\left(x_{i}\right) & =\prod_{k=1, k \neq i, j}^{N}\left(x_{i}-x_{k}\right), \\
M^{(2)}\left(x_{i}\right) & =N^{(2)}\left(x_{i}, x_{k}\right) \cdot\left(x_{i}-x_{k}\right)+2 N^{(1)}\left(x_{i}, x_{k}\right), \\
N^{(m)}\left(x_{i}, x_{j}\right) & =\frac{m\left[c_{i i}^{(m-1)} M^{(1)}\left(x_{i}\right)-c_{i j}^{(m-1)} M^{(1)}\left(x_{j}\right)\right]}{x_{i}-x_{j}} .
\end{aligned}
$$




\section{S. A. Ahmadi et al. / Applied and Computational Mechanics 13 (2019) 5-20}

For the second order derivations, we have:

$$
\left.\frac{\partial^{2} f}{\partial x^{2}}\right|_{x=x_{i}}=\sum_{j=1}^{Q} \sum_{k=1}^{Q} c_{i k}^{(1)} \cdot c_{k j}^{(1)} f\left(r, x_{j}\right) .
$$

In terms of differential quadrature, the discretized form of buckling equations of face sheets at all grid points $(i=2, \ldots, N-1, j=2, \ldots, Q-1)$ can be obtained as follows:

$$
\begin{aligned}
& C_{11}^{(i, o)} \sum_{k=1}^{N} a_{i k}^{(2)} B_{k j}+\frac{C_{11}^{(i, o)}}{r} \sum_{k=1}^{N} a_{i k}^{(1)} B_{k j}+C_{55}^{(i, o)} \sum_{k=1}^{Q} c_{j k}^{(2)} B_{i k}+ \\
& \left(C_{13}^{(i, o)}+C_{55}^{(i, o)}\right) \sum_{k=1}^{N} \sum_{l=1}^{Q} a_{i k}^{(1)} c_{j l}^{(1)} C_{k l}-\frac{\left(C_{22}^{(i, o)}+C_{66}^{(i, o)} \cdot m^{2}\right)}{r^{2}} B_{i j}+ \\
& \frac{C_{12}^{(i, o)}+C_{66}^{(i, o)}}{r} \sum_{k=1}^{N} a_{i k}^{(1)} A_{k j}-\frac{m\left(C_{22}^{(i, o)}+C_{66}^{(i, o)}\right)}{r^{2}} A_{i j}+ \\
& p\left[\frac{\mathrm{d} f_{r r}}{\mathrm{~d} r} \sum_{k=1}^{N} a_{i k}^{(1)} B_{k j}+f_{r r} \sum_{k=1}^{N} a_{i k}^{(2)} B_{k j}+\frac{f_{r r}}{r} \sum_{k=1}^{N} a_{i k}^{(1)} B_{k j}-\frac{2 m f_{\theta \theta}}{r^{2}} A_{i j}-\frac{\left(m^{2}+1\right) f_{\theta \theta}}{r^{2}} B_{i j}\right]=0, \\
& C_{66}^{(i, o)} \sum_{k=1}^{N} a_{i k}^{(2)} A_{k j}-\frac{m\left(C_{66}^{(i, o)}+C_{12}^{(i, o)}\right)}{r} \sum_{k=1}^{N} a_{i k}^{(1)} B_{k j}-\frac{m\left(C_{66}^{(i, o)}+C_{22}^{(i, o)}\right)}{r^{2}} B_{i j}+ \\
& \frac{C_{66}^{(i, o)}}{r} \sum_{k=1}^{N} a_{i k}^{(1)} A_{k j}-\frac{\left(C_{66}^{(i, o)}+m^{2}\left(C_{22}^{(i, o)}\right)\right)}{r^{2}} A_{i j}+ \\
& C_{44}^{(i, o)} \sum_{k=1}^{Q} c_{j k}^{(2)} A_{i k}-\frac{m\left(C_{44}^{(i, o)}+C_{23}^{(i, o)}\right)}{r} \sum_{k=1}^{Q} c_{j k}^{(1)} C_{i k}+ \\
& P\left[\frac{\mathrm{d} f_{r r}}{\mathrm{~d} r} \sum_{k=1}^{N} a_{i k}^{(1)} A_{k j}+f_{r r} \sum_{k=1}^{N} a_{i k}^{(2)} A_{k j}+\frac{f_{r r}}{r} \sum_{k=1}^{N} a_{i k}^{(1)} A_{k j}-\frac{2 m f_{\theta \theta}}{r^{2}} B_{i j}-\frac{\left(m^{2}+1\right) f_{\theta \theta}}{r^{2}} A_{i j}\right]=0 \\
& C_{33}^{(i, o)} \sum_{k=1}^{Q} c_{j k}^{(2)} C_{i k}+C_{55}^{(i, o)} \sum_{k=1}^{N} a_{i k}^{(2)} C_{k j}+\frac{C_{55}^{(i, o)}}{r} \sum_{k=1}^{N} a_{i k}^{(1)} C_{k j}+ \\
& \left(C_{55}^{(i, o)}+C_{13}^{(i, o)}\right) \sum_{k=1}^{N} \sum_{l=1}^{Q} a_{i k}^{(1)} c_{j l}^{(1)} B_{k l}+\frac{\left.C_{55}^{(i, o)}+C_{23}^{(i, o)}\right)}{r} \sum_{k=1}^{Q} c_{j k}^{(1)} B_{i k}-\frac{G m^{2}}{r^{2}} C_{i j}+ \\
& \frac{m\left(C_{66}^{(i, o)}+C_{23}^{(i, o)}\right)}{r} \sum_{k=1}^{Q} c_{j k}^{(1)} A_{i k}+\sigma_{\theta \theta}^{0} \frac{m^{2}}{r^{2}} C_{i j}+ \\
& P\left[\frac{\mathrm{d} f_{r r}}{\mathrm{~d} r} \sum_{k=1}^{N} a_{i k}^{(1)} C_{k j}+f_{r r} \sum_{k=1}^{N} a_{i k}^{(2)} C_{k j}+f_{r r} \sum_{k=1}^{N} a_{i k}^{(2)} C_{k j}-\frac{m^{2} f_{\theta \theta}}{r^{2}} C_{i j}+\frac{f_{r r}}{r} \sum_{k=1}^{N} a_{i k}^{(1)} C_{k j}\right]=0 .
\end{aligned}
$$

Here $C_{i j}^{(i, o)}$ denoting the stiffness constants of inner and outer face sheets [11]. Also the discrete form of the governing equations for the core can be written as:

$$
\begin{array}{r}
(2 G+\lambda) \sum_{k=1}^{N} a_{i k}^{(2)} B_{k j}+\frac{(2 G+\lambda)}{r} \sum_{k=1}^{N} a_{i k}^{(1)} B_{k j}+G \sum_{k=1}^{Q} c_{j k}^{(2)} B_{i k}+ \\
(G+\lambda) \sum_{k=1}^{N} \sum_{l=1}^{Q} a_{i k}^{(1)} c_{j l}^{(1)} B_{k l}-\frac{\left(2 G+\lambda+G \cdot m^{2}\right)}{r^{2}} B_{i j}+
\end{array}
$$




$$
\begin{gathered}
\frac{m(G+\lambda)}{r} \sum_{k=1}^{N} a_{i k}^{(1)} A_{k j}-\frac{m(3 G+\lambda)}{r^{2}} A_{i j}+ \\
p\left[\frac{\mathrm{d} f_{r r}}{\mathrm{~d} r} \sum_{k=1}^{N} a_{i k}^{(1)} B_{k j}+f_{r r} \sum_{k=1}^{N} a_{i k}^{(2)} B_{k j}+\frac{f_{r r}}{r} \sum_{k=1}^{N} a_{i k}^{(1)} B_{k j}-\frac{2 m f_{\theta \theta}}{r^{2}} A_{i j}-\frac{\left(m^{2}+1\right) f_{\theta \theta}}{r^{2}} B_{i j}\right]=0, \\
G \sum_{k=1}^{N} a_{i k}^{(2)} A_{k j}-\frac{m(G+\lambda)}{r} \sum_{k=1}^{N} a_{i k}^{(1)} B_{k j}-\frac{m(3 G+\lambda)}{r^{2}} B_{i j}+G(z) \sum_{k=1}^{Q} c_{j k}^{(2)} A_{i k}- \\
\frac{\left(G+m^{2}(2 G+\lambda)\right)}{r^{2}} A_{i j}+\frac{G(z)}{r} \sum_{k=1}^{N} a_{i k}^{(1)} A_{k j}-\frac{m(G+\lambda)}{r} \sum_{k=1}^{Q} c_{j k}^{(1)} C_{i k}-\sigma_{\theta \theta}^{0} \frac{2 m}{r^{2}} B_{i j}+ \\
\frac{\mathrm{d} f_{r r}}{\mathrm{~d} r} \sum_{k=1}^{N} a_{i k}^{(1)} A_{k j}+f_{r r} \sum_{k=1}^{N} a_{i k}^{(2)} A_{k j}+\frac{f_{r r}}{r} \sum_{k=1}^{N} a_{i k}^{(1)} C_{k j}^{(1)}+\frac{m(G+\lambda)}{r} \sum_{k j}-\frac{2 m f_{\theta \theta}}{r^{2}} B_{i j}-\frac{\left(m^{2}+1\right) f_{\theta \theta}}{r^{2}} A_{i j}^{(1)} A_{i k}+(G+\lambda) \sum_{k=1}^{N} \sum_{l=1}^{Q} a_{i k}^{(1)} c_{j l}^{(1)} B_{k l}+ \\
G \sum_{k=1}^{N} a_{i k}^{(2)} C_{k j}+(2 G+\lambda) \sum_{k=1}^{Q} c_{j k}^{(2)} A_{i k}-\frac{G m^{2}}{r^{2}} C_{i j}+\frac{(G+\lambda)}{r} \sum_{k=1}^{Q} c_{j k}^{(1)} B_{i k}+ \\
P\left[\frac{\mathrm{d} f_{r r}}{\mathrm{~d} r} \sum_{k=1}^{N} a_{i k}^{(1)} C_{k j}+f_{r r} \sum_{k=1}^{N} a_{i k}^{(2)} C_{k j}+f_{r r} \sum_{k=1}^{N} a_{i k}^{(2)} C_{k j}-\frac{m^{2} f_{\theta \theta}}{r^{2}} C_{i j}+\frac{f_{r r}}{r} \sum_{k=1}^{N} a_{i k}^{(1)} C_{k j}\right]=0 .
\end{gathered}
$$

In addition, we have to rewrite the boundary conditions in the discrete form. As an example, the boundary conditions (12) have been presented in the discrete form as follows:

$$
\begin{aligned}
C_{11}^{(i, o)} \sum_{k=1}^{N} a_{i k}^{(1)} B_{k j}+C_{13}^{(i, o)} \sum_{k=1}^{Q} c_{i k}^{(1)} C_{k j}+\frac{C_{12}^{(i, o)}}{r}\left(B_{i j}+m \cdot A_{i j}\right)=0 \quad i=1, N, \\
C_{66}^{(i, o)} \sum_{k=1}^{N} a_{i k}^{(1)} A_{k j}-\frac{C_{66}^{(i, o)}}{r}\left(A_{i j}+m \cdot B_{i j}\right)=0 \quad i=1, N, \\
C_{55}^{(i, o)}\left(\sum_{k=1}^{Q} c_{j k}^{(1)} B_{i k}+\sum_{k=1}^{N} a_{i k}^{(1)} C_{k j}\right)=0 \quad i=1, N .
\end{aligned}
$$

It is clear that we have $3 \times N \times Q$ algebraic equations at boundary and domain grid points. The value of the buckling pressure is obtained by solving the standard eigenvalue equation presented in the partitioned form as:

$\left(-[\mathbf{D B G}][\mathbf{B B}]^{-1}[\mathbf{B D}]+[\mathbf{D D G}]\right)^{-1}\left(-[\mathbf{D B}][\mathbf{B B}]^{-1}[\mathbf{B D}]+[\mathbf{D D}]\right)[u v w]^{T}-P[\mathbf{I}][u v w]^{T}=0$,

where sub-matrices $[\mathbf{B B}],[\mathrm{BD}]$ are obtained from the boundary conditions and $[\mathbf{D B G}],[\mathbf{D D}]$, $[\mathrm{DB}],[\mathrm{DDG}]$ are resulted from the discrete governing equations. It is clear that equation (25) can be treated as a conventional eigenvalue problem to obtain the buckling pressure and the corresponding mode shapes of the system.

\section{Results and discussion}

In order to illustrate the effectiveness of presented formulations and solution method for buckling analysis of thick sandwich cylindrical shells by considering the face sheets imperfections, the 


\section{S. A. Ahmadi et al. / Applied and Computational Mechanics 13 (2019) 5-20}

results are compared against results reported in the literature. It is assumed that the face sheets consist of orthotropic materials like that Carbon/epoxy (Case 1) and Glass/epoxy (Case 2). In this study inner radius of sandwich is $R_{1}=1 \mathrm{~m}$, total thickness of shell is $h=R_{2}-R_{1}$ and core is made of isotropic alloy foam and has the same properties for two cases of shells. Material properties for the face sheets and the core are given in Table 1. It should be mentioned that the shells are simply supported at two ends and are under the action of uniform lateral external pressure. The normalized results of buckling pressures for a thick monolithic orthotropic cylindrical shell are presented in Tables 2 and 3 for cases 1 and 2, respectively. The results have been compared with those reported in references $[12,14,28]$ in which an intact shell has been studied. Furthermore, the accuracy of the presented method for thick sandwich orthotropic shell (Case 1) is validated against results obtained by finite element software ANSYS and those reported by [14] in Figs. 2 and 3. This analysis is used to predict the bifurcation point on F-Ugraph using linearized model of elastic structure. Quadrilateral shell elements, namely Shell281, with eight nodes are used to model the thick sandwich cylindrical shell. In order to find the buckling mode number, an exhaustive study was made for the $m$ and $n$ that results in

Table 1. Properties of materials employed in the present study

\begin{tabular}{|c|ccccccccc|}
\hline Material & $\begin{array}{c}E_{11} \\
(\mathrm{GPa})\end{array}$ & $\begin{array}{c}E_{22} \\
(\mathrm{GPa})\end{array}$ & $\begin{array}{c}E_{33} \\
(\mathrm{GPa})\end{array}$ & $\begin{array}{c}G_{12} \\
(\mathrm{GPa})\end{array}$ & $\begin{array}{c}G_{31} \\
(\mathrm{GPa})\end{array}$ & $\begin{array}{c}G_{23} \\
(\mathrm{GPa})\end{array}$ & $\nu_{12}$ & $\nu_{23}$ & $\nu_{13}$ \\
\hline Carbon/epoxy & 10.3 & 181 & 10.3 & 7.17 & 5.96 & 7.17 & 0.068 & 0.227 & 0.4 \\
Glass/epoxy & 14 & 57 & 14 & 5.7 & 5 & 5.7 & 0.016 & 0.28 & 0.49 \\
Alloy foam Core & 0.046 & 0.046 & 0.046 & 0.017 & 0.017 & 0.017 & 0.33 & 0.33 & 0.33 \\
\hline
\end{tabular}

Table 2. Comparison of normalized buckling pressure of a thick monolithic orthotropic shell (Case 2), $L / R_{2}=10 \tilde{P}=P R_{2}^{3} / E_{2} h^{3}, m=2, n=1$

\begin{tabular}{|cccc|}
\hline$R_{2} / R_{1}$ & Present study & {$[12]$} & {$[28]$} \\
\hline 1.05 & 0.2712 & 0.2813 & - \\
1.10 & 0.2459 & 0.2744 & 0.2663 \\
1.15 & 0.2407 & 0.2758 & 0.2657 \\
1.20 & 0.2362 & 0.2764 & 0.2635 \\
1.25 & 0.2318 & 0.2755 & 0.2572 \\
1.30 & 0.2245 & 0.2733 & 0.2510 \\
\hline
\end{tabular}

Table 3. Comparison of the buckling pressure $(\mathrm{kPa})$ of a thick monolithic orthotropic shell (Case 1), $L / R_{2}=20, m=2, n=1$

\begin{tabular}{|ccc|}
\hline$R_{2} / R_{1}$ & Present study & {$[14]$} \\
\hline 1.10 & 0.37 & 0.25 \\
1.15 & 1.10 & 1.00 \\
1.20 & 1.70 & 1.80 \\
1.25 & 2.75 & 2.80 \\
1.30 & 3.90 & 4.10 \\
1.35 & 5.10 & 5.16 \\
1.40 & 6.30 & 6.20 \\
\hline
\end{tabular}




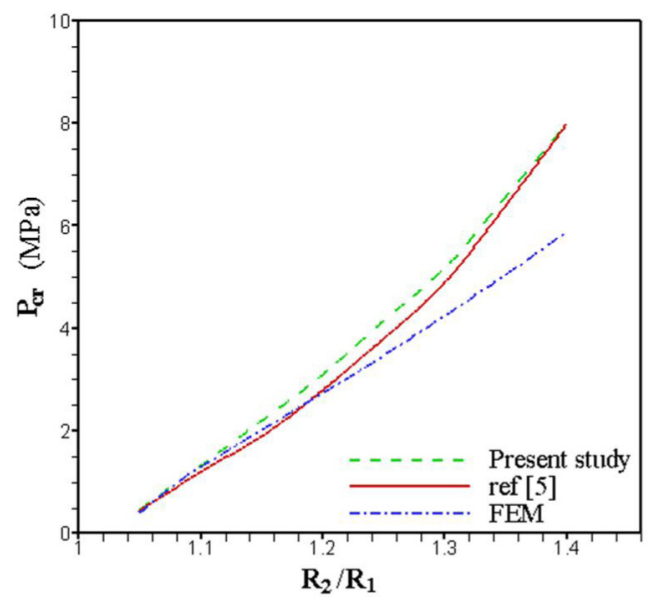

Fig. 2. Comparison of the buckling pressure $(\mathrm{kPa})$ of an intact thick composite sandwich shell (Case 1), $L / a=20$

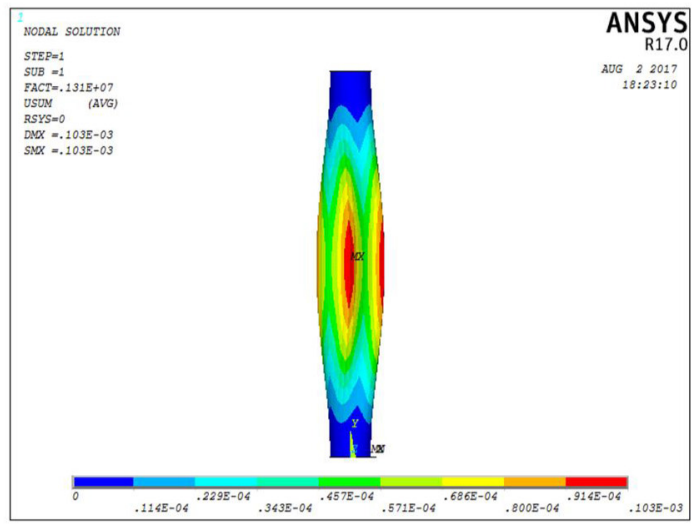

a

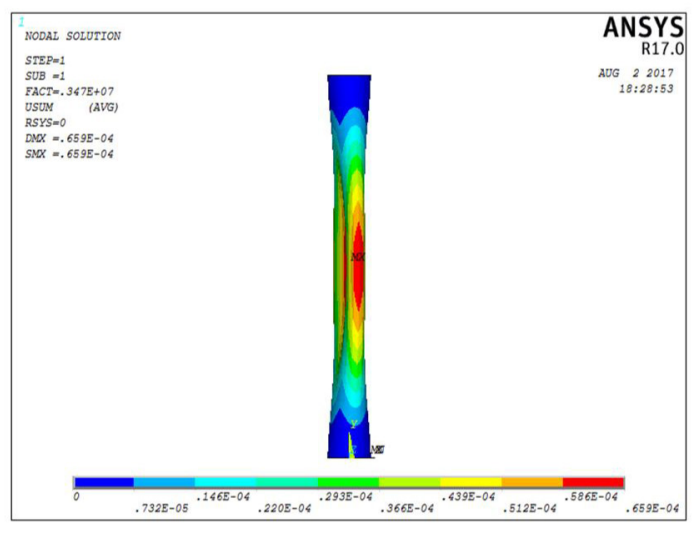

$\mathrm{c}$

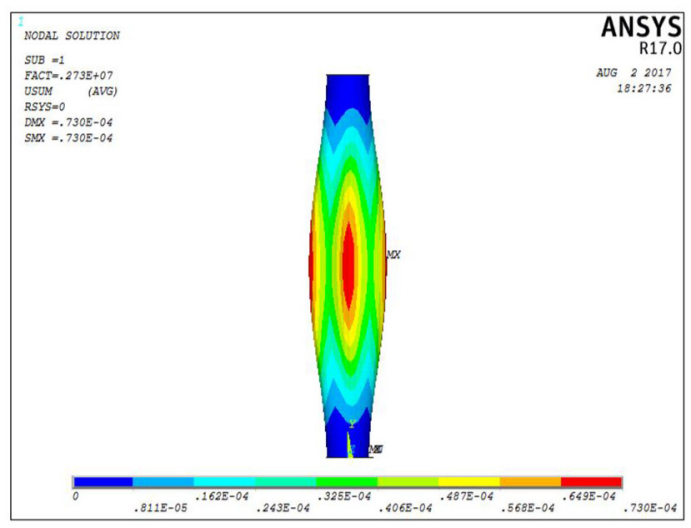

b

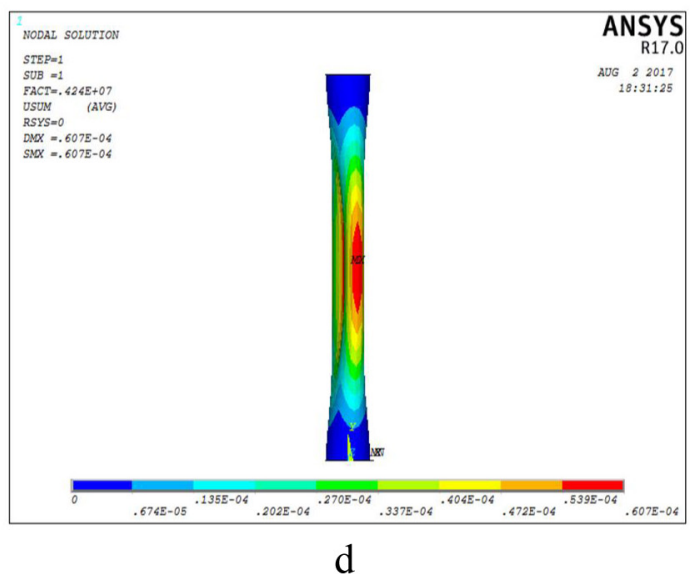

Fig. 3. The buckling pressure $(\mathrm{kPa})$ and the corresponding mode shape of an intact thick sandwich shell (Case 1), $L / a=20$ : a) $R_{2} / R_{1}=1.1$, b) $R_{2} / R_{1}=1.2$, c) $R_{2} / R_{1}=1.25$, d) $R_{2} / R_{1}=1.3$

the minimum eigenvalue. It was found that for these panels, $m=2$ and $n=1$ corresponds to the lowest eigenvalue. It is concluded that from these figure and tables that the critical buckling pressure increases exponentionaly as the outer-to-inner radius ratio of panels increases. It can be seen that the results of presented method for thick sandwich orthotropic shells are in good agreements with those reported in the literatures and also with ANSYS. 
Next, the buckling behavior of a thick sandwich orthotropic shell is investigated. Variation of the critical buckling pressure versus the ratio of outer to inner radius for the sandwich shell with $L / R_{2}=5$ and $h_{i}=h_{o}=0.1 h, h_{c}=0.8 h, h=R_{2}-R_{1}$ is presented in Table 4 . It can be seen from Table 4 that as the thickness of the shell increases the variation of the buckling pressure increase with the imperfection parameter. Moreover, buckling pressure and the corresponding buckling mode shapes obtained by finite element software ANSYS are shown in Fig. 4. To illustrate the effect of material constants and thickness ratio of core and face sheets, Table 5 shows the buckling pressure for various ratios of outer to inner radius $R_{2} / R_{1}$ and length to outer radius $L / R_{2}$.

Table 4. Buckling pressure $P_{c r}(\mathrm{kPa})$ of sandwich shells

\begin{tabular}{|c|cccc|}
\hline \multirow{2}{*}{$R_{2} / R_{1}$} & \multicolumn{2}{|c}{ DQM } & \multicolumn{2}{c|}{ FEM } \\
\cline { 2 - 5 } & Case 1 & Case 2 & Case 1 & Case 2 \\
\hline 1.05 & 0.775 & 0.665 & 0.77 & 0.658 \\
1.10 & 1.80 & 1.50 & 1.64 & 1.40 \\
1.15 & 2.90 & 2.43 & 2.43 & 2.21 \\
1.20 & 4.35 & 3.24 & 3.75 & 3.16 \\
1.25 & 5.80 & 4.20 & 5.57 & 4.05 \\
1.30 & 7.22 & 5.30 & 6.98 & 5.12 \\
1.35 & 8.83 & 5.80 & 8.61 & 5.72 \\
1.40 & 10.80 & 7.70 & 10.67 & 7.56 \\
\hline
\end{tabular}

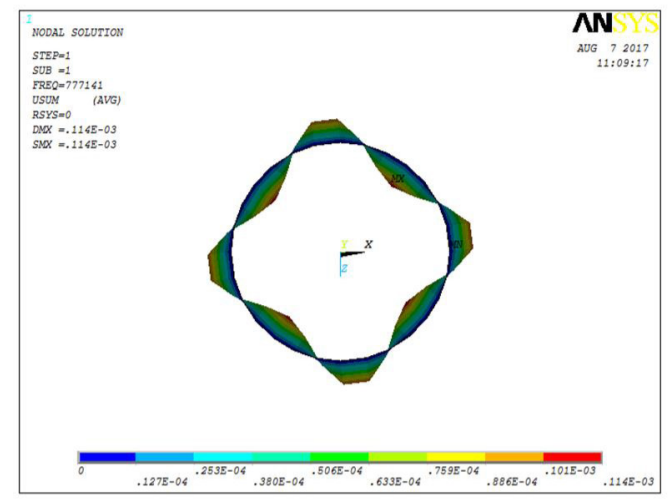

a

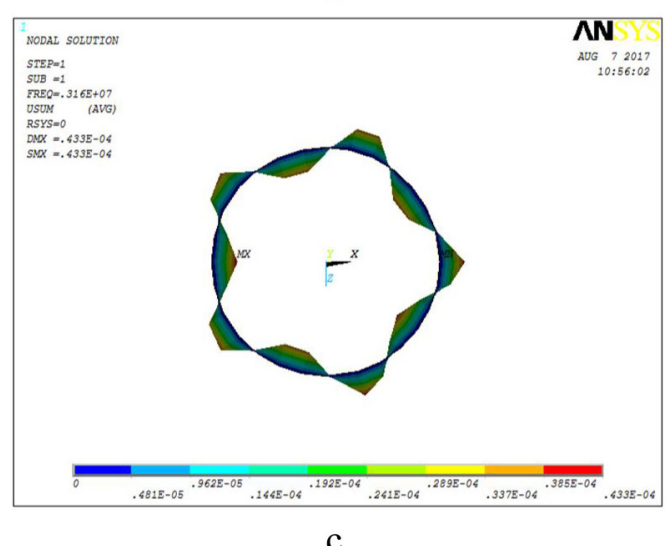

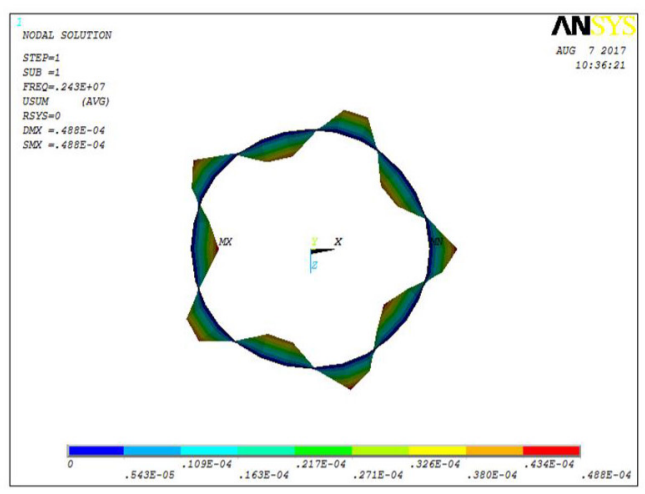

b

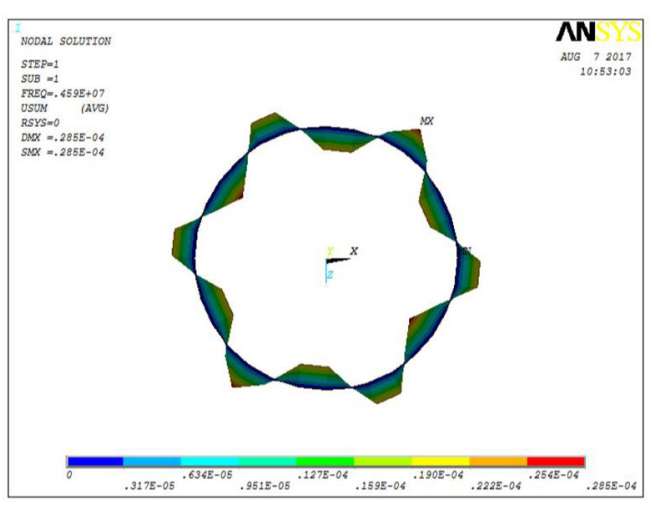

d

Fig. 4. Buckling pressure and mode shapes of thick sandwich shells, $L / a=5$ : a) $R_{2} / R_{1}=1.05$ (Case 1 ), b) $R_{2} / R_{1}=1.15$ (Case 1), c) $R_{2} / R_{1}=1.2$ (Case 2), d) $R_{2} / R_{1}=1.3$ (Case 2) 
Table 5. Buckling pressure $P_{c r}(\mathrm{kPa})$ of sandwich shells

\begin{tabular}{|c|c|c|c|c|c|}
\hline \multirow{2}{*}{\multicolumn{2}{|c|}{$L / R_{2}$}} & \multicolumn{2}{|c|}{ Case 1} & \multicolumn{2}{|c|}{ Case 2} \\
\hline & & $R_{2} / R_{1}=1.05$ & $R_{2} / R_{1}=1.2$ & $R_{2} / R_{1}=1.05$ & $R_{2} / R_{1}=1.2$ \\
\hline \multirow{3}{*}{3} & $h_{i}=h_{o}=0.05 h, h_{c}=0.9 h$ & 0.787 & 3.30 & 0.710 & 2.90 \\
\hline & $h_{i}=h_{o}=0.1 h, h_{c}=0.8 h$ & 0.870 & 4.99 & 0.744 & 3.84 \\
\hline & $h_{i}=h_{o}=0.2 h, h_{c}=0.6 h$ & 1.450 & 17.02 & 1.020 & 7.49 \\
\hline \multirow{3}{*}{5} & $h_{i}=h_{o}=0.05 h, h_{c}=0.9 h$ & 0.676 & 2.89 & 0.446 & 1.96 \\
\hline & $h_{i}=h_{o}=0.1 h, h_{c}=0.8 h$ & 0.775 & 4.35 & 0.666 & 3.24 \\
\hline & $h_{i}=h_{o}=0.2 h, h_{c}=0.6 h$ & 1.110 & 9.87 & 0.815 & 6.10 \\
\hline \multirow{3}{*}{20} & $h_{i}=h_{o}=0.05 h, h_{c}=0.9 h$ & 0.625 & 2.10 & 0.420 & 1.85 \\
\hline & $h_{i}=h_{o}=0.1 h, h_{c}=0.8 h$ & 0.670 & 3.17 & 0.486 & 2.40 \\
\hline & $h_{i}=h_{o}=0.2 h, h_{c}=0.6 h$ & 0.733 & 6.98 & 0.527 & 4.01 \\
\hline
\end{tabular}

To study the effects of geometrical imperfections on the stability of sandwich shells, a quantity namely, $\lambda_{p}$ denoting the ratios of the buckling loads of the imperfect shell to the intact shell in various load conditions are introduced as:

$$
\lambda_{p}=\frac{P_{c r}^{(\mathrm{imper})}}{P_{c r}^{(\text {per })}} .
$$

Variation of the buckling pressure reduction parameter $\lambda_{p}$ of imperfect sandwich orthotropic shells with various outer to inner radius ratio and same ratio of length to outer radius $L / R_{2}=1$ with $h_{i}=h_{o}=0.1 h, h_{c}=0.8 h$ are presented in Tables 6,7 and 8 . It should be noted that the

Table 6. Buckling pressure reduction parameter $\lambda_{P}$ of shells with various $R_{2} / R_{1}$ ratios under pure lateral loading for various imperfection factors of inner face sheet (Case 1)

\begin{tabular}{|c|cccccc|}
\hline \multirow{2}{*}{$\varepsilon_{1}$} & \multicolumn{6}{|c|}{$R_{2} / R_{1}$} \\
\cline { 2 - 7 } & 1.05 & 1.10 & 1.15 & 1.20 & 1.30 & 1.40 \\
\hline 0.00 & 1.000 & 1.000 & 1.000 & 1.000 & 1.000 & 1.000 \\
0.02 & 0.995 & 0.987 & 0.975 & 0.971 & 0.968 & 0.945 \\
0.05 & 0.982 & 0.961 & 0.953 & 0.940 & 0.909 & 0.898 \\
0.10 & 0.977 & 0.943 & 0.922 & 0.906 & 0.831 & 0.822 \\
0.15 & 0.967 & 0.911 & 0.888 & 0.872 & 0.777 & 0.767 \\
0.20 & 0.959 & 0.885 & 0.859 & 0.828 & 0.720 & 0.704 \\
\hline
\end{tabular}

Table 7. Buckling pressure reduction parameter $\lambda_{P}$ of shells with various $R_{2} / R_{1}$ ratios under pure lateral loading for various imperfection factors of outer face sheet (Case 1)

\begin{tabular}{|c|cccccc|}
\hline \multirow{2}{*}{$\varepsilon_{2}$} & \multicolumn{6}{|c|}{$R_{2} / R_{1}$} \\
\cline { 2 - 7 } & 1.05 & 1.10 & 1.15 & 1.20 & 1.30 & 1.40 \\
\hline 0.00 & 1.000 & 1.000 & 1.000 & 1.000 & 1.000 & 1.000 \\
0.02 & 0.997 & 0.984 & 0.981 & 0.980 & 0.975 & 0.964 \\
0.05 & 0.988 & 0.974 & 0.969 & 0.949 & 0.925 & 0.905 \\
0.10 & 0.982 & 0.948 & 0.934 & 0.917 & 0.874 & 0.851 \\
0.15 & 0.971 & 0.917 & 0.909 & 0.885 & 0.819 & 0.796 \\
0.20 & 0.966 & 0.893 & 0.882 & 0.850 & 0.766 & 0.723 \\
\hline
\end{tabular}


Table 8. Buckling pressure reduction parameter $\lambda_{P}$ of shells with various $R_{2} / R_{1}$ ratios under pure lateral loading for various imperfection factors of two face sheets (Case 1)

\begin{tabular}{|c|cccccc|}
\hline \multirow{2}{*}{$\varepsilon_{1}, \varepsilon_{2}$} & \multicolumn{6}{|c|}{$R_{2} / R_{1}$} \\
\cline { 2 - 7 } & 1.05 & 1.10 & 1.15 & 1.20 & 1.30 & 1.40 \\
\hline 0.00 & 1.000 & 1.000 & 1.000 & 1.000 & 1.000 & 1.000 \\
0.02 & 0.979 & 0.969 & 0.956 & 0.940 & 0.923 & 0.905 \\
0.05 & 0.950 & 0.921 & 0.891 & 0.880 & 0.855 & 0.850 \\
0.10 & 0.883 & 0.854 & 0.825 & 0.800 & 0.737 & 0.695 \\
0.15 & 0.811 & 0.781 & 0.750 & 0.707 & 0.660 & 0.576 \\
0.20 & 0.762 & 0.729 & 0.709 & 0.641 & 0.609 & 0.545 \\
\hline
\end{tabular}

thickness of the core is assumed to be constant and face sheets thickness varied axisymmetrically in radial directions. It can be concluded that imperfections which can be caused due to manufacturing process or service life have significant effects on the buckling load of shells. It is also revealed that the geometrical imperfections have higher effects on the buckling load of thick shells than thin ones. The results have shown that outer face sheet plays a more important role in the stability of the sandwich shell than the inner layer. For the same imperfection parameter in two face sheets, buckling pressure reduction for the outer face sheet is higher than inner ones. Also, It is found that the buckling pressure reduction parameter has almost linear variation with the ratio of the outer-to-inner radius of the shell.

The effect of the ratio of the length to the outer radius, i.e., $L / R_{2}$ on the critical pressure of sandwich orthotropic cylindrical shell with $R_{2} / R_{1}=1.15$ and various imperfection parameters $\varepsilon_{1}=\varepsilon_{2}$, is shown in Fig. 5. It is realized from this figure that as the ratio of $L / R_{2}$ increases, the buckling load decreases and approaches to an asymptotic value. These values can be considered for buckling pressure of long sandwich orthotropic shells.

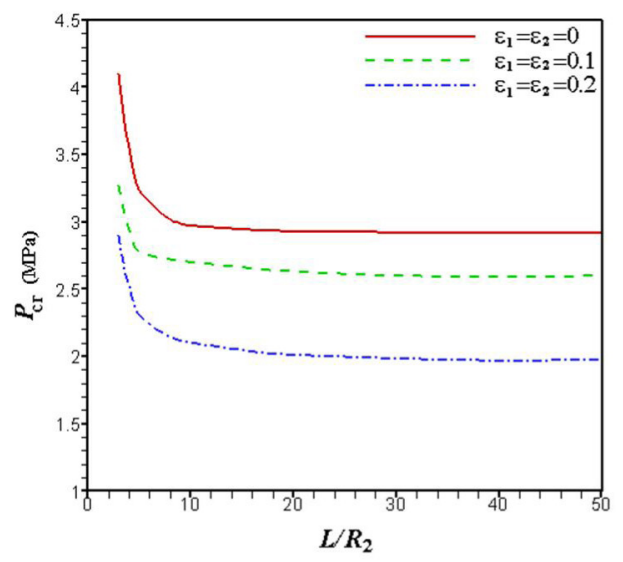

a)

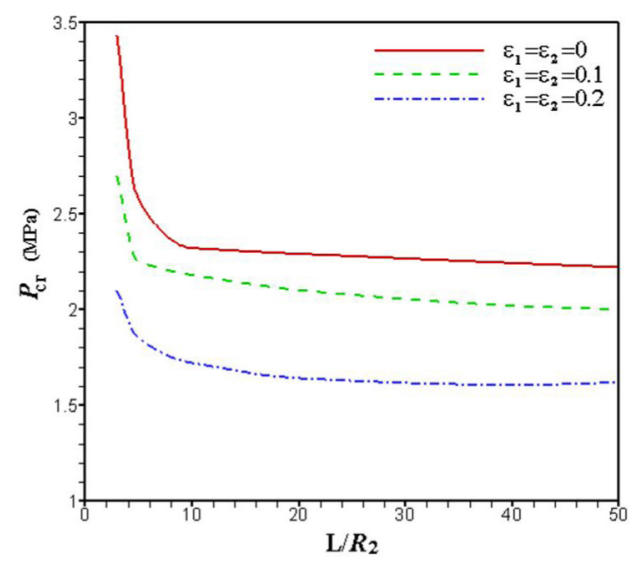

b)

Fig. 5. Variation of buckling external pressure (MPa) of a thick imperfect sandwich orthotropic shell versus $L / R_{2}$ : a) Case $1, \mathrm{~b}$ ) Case 2

\section{Conclusion}

In this paper, the differential quadrature method is applied to calculate the buckling lateral pressure of thick sandwich orthotropic cylindrical shells with geometrical imperfections. It is assumed that core thickness is constant and the face sheets have an axisymmetrical varied 


\section{S. A. Ahmadi et al. / Applied and Computational Mechanics 13 (2019) 5-20}

thickness in the axial direction represented by a parameter (imperfection factor $\varepsilon$ ). Three dimensional buckling equations are taken in terms of the second Piola-Kirchhoff stress tensor using Benchmark three-dimensional elasticity solution. Two types of shells with the same core materials consist of alloy foam and different face sheets material, Carbon/epoxy and Glass/epoxy are studied. Numerical results for buckling pressure of sandwich shells and buckling pressure reduction factor $\left(\lambda_{P}\right)$ of these cases have been obtained. Deliberation of buckling pressure reduction factor indicates that the thick sandwich orthotropic shells are sensitive to geometrical imperfections more than thin ones.

\section{References}

[1] Akbari, A.R., Ahmadi, S. A., Buckling of imperfect thick cylindrical shells and curved panels with different boundary conditions under external pressure, Journal of Theoretical and Applied Mechanics 52 (1) (2014) 25-36.

[2] Arjomandi, K., Taheri, F., Elastic buckling capacity of bonded and unbonded sandwich pipes under external hydrostatic pressure, Journal of Mechanics of Materials and Structures 5 (3) (2010) 391-407.

[3] Arjomandi, K., Taheri, F., Stability and post-buckling response of sandwich pipes under hydrostatic external pressure, International Journal of Pressure Vessels and Piping 88 (2011) 138-148. https://doi.org/10.1016/j.ijpvp.2011.02.002

[4] Bellman, R. E., Casti, J., Differential quadrature and long term integration, Journal of Mathematical Analysis and Applications 34 (1) (1971) 235-238.

[5] Bert, C.W, Malik, M., The differential quadrature method for irregular domains and application to plate vibration, International Journal of Mechanical Sciences 38 (6) (1996) 589-606.

[6] Birman, V., Simitses, G., Elliptical and circular cylindrical sandwich shells with different facings, Journal of Sandwich Structures and Materials 2 (2000) 152-176. https://doi.org/10.1177/109963620000200204

[7] Ciarlet, P. G., Mathematical elasticity, Vol. I, Three-dimensional elasticity. North-Holland, Amsterdam, 1988.

[8] Degenhardt, R., DESICOS. DESICOS. European Union 7th Framework, Web. 06 Dec. 2012. http://www.desicos.eu.

[9] Degenhardt, R., Kling, A., Bethge, A., Orf, J., Kärger, L., Rohwer, K., Investigations of imperfection sensitivity of unstiffened CFRP cylindrical shells, Composite Structures 92 (2010) 1939-1946. https://doi.org/10.1016/j.compstruct.2009.12.014

[10] Donnell, L. H., A new theory for the buckling of thin cylinders under axial compression and bending. Transactions of the American Society of Mechanical Engineers 56 (AER-56-12) (1934) 795-806.

[11] Jones, R. M., Mechanics of composite materials, Taylor and Francis, Philadelphia, USA, 1999.

[12] Kardomateas, G. A., Buckling of thick orthotropic cylindrical shells under external pressure, ASME Journal of Applied Mechanics 60 (1993) 195-202. https://doi.org/10.1115/1.2900745

[13] Kardomateas, G. A., Elasticity solutions for a sandwich orthotropic cylindrical shell under external pressure, internal pressure and axial force, AIAA Journal 39 (4) (2001) 713-719.

[14] Kardomateas, G. A., Elasticity solutions for the buckling of thick composite and sandwich cylindrical shells under external pressure, In: Daniel, I. M., Gdoutos, E. E., Rajapakse, Y. D. S. (Eds.) Major accomplishments in composite materials and sandwich structures, Springer, Dordrecht, 2009.

[15] Kardomateas, G. A., Simitses, G. J., Buckling of long sandwich cylindrical shells under external pressure, Journal of Applied Mechanics (ASME) 72 (4) (2005) 493-499. 
[16] Koiter, W. T., On the stability of elastic equilibrium. NASA TT-F-10833, National Aeronautics and Space Administration, USA, 1967.

[17] Lai, W. M., Rubin, D., Krempl, E., Introduction to continuum mechanics, 3rd ed. ButterworthHeinemann, Massachusetts, 1996.

[18] Lekhnitskii, S. G., Theory of elasticity of an anisotropic elastic body, Holden Day, San Francisco, also Mir, Moscow, 1981.

[19] Lopatin, A.V., Morozov, E.V., Buckling of the composite sandwich cylindrical shell with clamped ends under uniform external pressure, Composite Structures 122 (2015) 209-216. https://doi.org/10.1016/j.compstruct.2014.11.048

[20] Lundquist, E. E., Strength tests of thin-walled duralumin cylinders in compression. NACA Report No. 473, 1933.

[21] Maleki, A., Ahmadi, S. A., Buckling analysis of orthotropic thick cylindrical shells considering geometrical imperfection using differential quadrature method (DQM), Journal of Theoretical and Applied Mechanics 48 (3) (2018) 45-60.

[22] Malikan, M., Sadraee Far, M. N., Differential quadrature method for dynamic buckling of graphene sheet coupled by a viscoelastic medium using neperian frequency based on nonlocal elasticity theory, Journal of Appllied and Computational Mechanics 4 (3) (2018) 147-160.

[23] Malikan, M., Tornabene, F., Dimitri, R., Nonlocal three-dimensional theory of elasticity for buckling behavior of functionally graded porous nanoplates using volume integrals, Materials Research Express 5 (9) (2018).

[24] NASA, NASA SP-8007 guideline, 1965.

[25] Ohga, M., Wijenayaka, A.S., Croll, J.G.A., Reduced stiffness buckling of sandwich cylindrical shells under uniform external pressure, Thin-Walled Structures 43 (2005) 1188-1201. https://doi.org/10.1016/j.tws.2005.03.006

[26] Orifici, A. C., Thomson, R. S., Degenhardt, R., Bisagni, C., Bayandor, J., An analysis tool for design and certification of postbuckling composite aerospace structures, International Journal of Structural Stability and Dynamics 10 (2010) 669-681. https://doi.org/10.1142/S0219455410003671

[27] Shu, C., Differential quadrature and its application in engineering, Springer-Verlag, London, UK, 2000.

[28] Soldatos, K. P., Jian-Qiao, Ye., Three-dimensional static, dynamic, thermo-elastic and buckling analysis of homogeneous and laminated composite cylinders, Composite Structures 29 (1994) 131-143. https://doi.org/10.1016/0263-8223(94)90095-7

[29] Wang, B., Shiyang, Zhua S., Hao, P., Bi, X., Du, K., Chen, B., Ma, X., Chao, Y. J., Buckling of quasi-perfect cylindrical shell under axial compression: A combined experimental and numerical investigation, International Journal of Solids and Structures 130-131 (2018) 232-247. https://doi.org/10.1016/j.ijsolstr.2017.09.029

[30] Wang, X., Wang, Y., Static analysis of sandwich panels with non-homogeneous soft cores by novel weak form quadrature element method, Composite Structures 146 (2016) 207-220. https://doi.org/10.1016/j.compstruct.2016.03.017

[31] Wang, X., Yuan, Zh., Accurate stress analysis of sandwich panels by the differential quadrature method, Applied Mathematical Modelling 43 (548) (2017) 548-565.

[32] Wang, Z., Han, Q., Nash, D. H., Liu, P., Hu, D., Investigation of imperfect effect on thermal buckling of cylindrical shell with FGM coating, European Journal of Mechanics / A Solids 69 (2018) 221-230. https://doi.org/10.1016/j.euromechsol.2018.01.004 